\title{
A Quantitative Variant of Voronovskaja's Theorem for King-Type Operators
}

\author{
ZOLTÁN FINTA*
}

ABSTRACT. In this note we establish a quantitative Voronovskaja theorem for modified Bernstein polynomials using the first order Ditzian-Totik modulus of smoothness.

Keywords: Bernstein operators, Voronovskaja theorem, King operators, First order Ditzian-Totik modulus of smoothness

2010 Mathematics Subject Classification: 41A25, 41A36.

\section{INTRODUCTION}

The Bernstein polynomials are defined by

$$
\left(B_{n} f\right)(x) \equiv B_{n}(f ; x)=\sum_{k=0}^{n} p_{n, k}(x) f\left(\frac{k}{n}\right),
$$

where $p_{n, k}(x)=\left(\begin{array}{l}n \\ k\end{array}\right) x^{k}(1-x)^{n-k}, x \in[0,1], f \in C[0,1]$ and $n \geq 1$. Among the properties of Bernstein polynomials we mention the following asymptotic formula, called Voronovskaja's theorem: if $f$ is bounded on $[0,1]$, differentiable in some neighborhood of $x \in[0,1]$, and has second derivative $f^{\prime \prime}(x)$, then

$$
\lim _{n \rightarrow \infty} n\left(\left(B_{n} f\right)(x)-f(x)\right)=\frac{1}{2} x(1-x) f^{\prime \prime}(x) .
$$

Further properties:

$$
\left(B_{n} e_{0}\right)(x)=1, \quad\left(B_{n} e_{1}\right)(x)=x \quad \text { and } \quad\left(B_{n} e_{2}\right)(x)=x^{2}+\frac{1}{n} x(1-x),
$$

where $e_{i}(x)=x^{i}, x \in[0,1]$ and $i \in\{0,1,2, \ldots\}$. In [8] King constructed a Bernstein-type operator, which preserves the functions $e_{0}$ and $e_{2}$. By modification of $f\left(\frac{k}{n}\right)$ in (1.1), Aldaz et al. [1] defined Bernstein-King-type operators possessing $e_{0}$ and $e_{j}$ as fixed points, where $j \in\{2,3, \ldots\}$ is arbitrary. These operators are given by

$$
\left(U_{n, j} f\right)(x) \equiv U_{n, j}(f ; x)=\sum_{k=0}^{n} p_{n, k}(x) f\left(a_{n, k}\right)
$$

(see [1, Proposition 11]), where $x \in[0,1], f \in C[0,1]$ and

$$
a_{n, k}=\sqrt[j]{\frac{k(k-1) \ldots(k-j+1)}{n(n-1) \ldots(n-j+1)}}, \quad n \geq j \geq 2 .
$$

The operators $U_{n, j}$ are linear and positive, $U_{n, j} e_{0}=e_{0}$ and $U_{n, j} e_{j}=e_{j}$, respectively.

Received: 13 April 2019; Accepted: 11 July 2019; Published Online: 13 July 2019

*Corresponding author: Z. Finta; fzoltan@math.ubbcluj.ro

DOI: $10.33205 / \mathrm{cma} .553427$ 
The goal of the paper is to obtain a quantitative Voronovskaja-type theorem for $U_{n, j}$ with the aid of the first order Ditzian-Totik modulus of smoothness defined by

$$
\omega_{\varphi}^{1}(f ; \delta)=\sup _{0<h \leq \delta} \sup _{x \pm \frac{1}{2} h \varphi(x)}\left|f\left(x+\frac{1}{2} h \varphi(x)\right)-f\left(x-\frac{1}{2} h \varphi(x)\right)\right|,
$$

where $\varphi(x)=\sqrt{x(1-x)}, x \in[0,1]$. It is known [2, Theorem 2.1.1] that (1.5) is equivalent with the $K$-functional

$$
K_{1, \varphi}(f ; \delta)=\inf _{g \in W(\varphi)}\left\{\|f-g\|+\delta\left\|\varphi g^{\prime}\right\|\right\}, \quad \delta>0,
$$

where $W(\varphi)=\left\{g \mid g \in A C_{l o c}[0,1],\left\|\varphi g^{\prime}\right\|<\infty\right\}$ and $g \in A C_{l o c}[0,1]$ means that $g$ is absolutely continuous in every closed interval $[a, b] \subseteq[0,1]$, i.e. there exists $C_{1}>0$ such that

$$
C_{1}^{-1} \omega_{\varphi}^{1}(f ; \delta) \leq K_{1, \varphi}(f ; \delta) \leq C_{1} \omega_{\varphi}^{1}(f ; \delta) .
$$

It is worth mentioning that Floater obtained a generalization of (1.2) in [4], dealing with the asymptotic behavior of differentiated Bernstein polynomials. Different quantitative versions of Floater's theorem were established in [5], [6], [7] and [3].

\section{MAIN RESULT}

In the sequel we need some lemmas.

Lemma 2.1. The inequalities $0 \leq 1-x^{n}-(1-x)^{n} \leq n x(1-x)$ hold true for all $x \in[0,1]$ and $n=1,2, \ldots$

Proof. For $x \in[0,1]$, we have

$$
x^{n}+(1-x)^{n} \leq x+(1-x)=1 .
$$

For the second inequality, we have

$$
\begin{gathered}
1-x^{n}-(1-x)^{n}=(1-x)\left(1+x+\ldots+x^{n-1}\right)-(1-x)^{n} \\
\quad=(1-x)\left[1+x+\ldots+x^{n-1}-(1-x)^{n-1}\right] \leq n x(1-x)
\end{gathered}
$$

iff $1+x+\ldots+x^{n-1} \leq n x+(1-x)^{n-1}$. We prove the former inequality by induction on $n$. If $n=1$, then $1 \leq x+1$; we suppose that $1+x+\ldots+x^{n-1} \leq n x+(1-x)^{n-1}$. Then, by (2.1),

$$
\begin{aligned}
1 & +x+\ldots+x^{n-1}+x^{n} \\
& \leq n x+(1-x)^{n-1}+x^{n}=(n+1) x+(1-x)^{n-1}-x+x^{n} \\
& =(n+1) x+(1-x)^{n}+(1-x)^{n-1}-(1-x)^{n}-x+x^{n} \\
& =(n+1) x+(1-x)^{n}+x(1-x)^{n-1}-x+x^{n} \\
& =(n+1) x+(1-x)^{n}-x\left(1-x^{n-1}-(1-x)^{n-1}\right) \leq(n+1) x+(1-x)^{n},
\end{aligned}
$$

which was to be proved.

Lemma 2.2. For the operator $U_{n, j}$ defined by (1.3)-(1.4) and $x \in[0,1]$, we have

a) $0 \leq U_{n, j}\left(x e_{0}-e_{1} ; x\right) \leq \frac{1}{n}(j-1)$;

b) $U_{n, j}\left(\left(e_{1}-x e_{0}\right)^{2} ; x\right) \leq \frac{2}{n}\left((j-1)^{2}+1\right) \varphi^{2}(x)$;

c) $U_{n, j}\left(\left(e_{1}-x e_{0}\right)^{4} ; x\right) \leq \frac{8}{n^{2}}\left((j-1)^{2}+1\right)$. 
Proof. Because $U_{n, j}$ is linear and preserves the functions $e_{0}$ and $e_{j}$, we obtain

$$
\begin{aligned}
U_{n, j}\left(x e_{0}-e_{1} ; x\right) & =x-U_{n, j}\left(e_{1} ; x\right)=\sum_{k=0}^{n} p_{n, k}(x) \frac{k}{n}-\sum_{k=0}^{n} p_{n, k}(x) a_{n, k} \\
& =\sum_{k=0}^{n} p_{n, k}(x)\left(\frac{k}{n}-a_{n, k}\right) .
\end{aligned}
$$

For $k \in\{j, j+1, \ldots, n\}$, we have $\frac{k-j+1}{n-j+1} \leq \ldots \leq \frac{k-1}{n-1} \leq \frac{k}{n}$. Hence

$$
\begin{aligned}
0 \leq \frac{k}{n}-a_{n, k} & \leq \frac{k}{n}-\frac{k-j+1}{n-j+1}=(j-1) \frac{n-k}{n(n-j+1)} \\
& \leq(j-1) \frac{n-j}{n(n-j+1)} \leq \frac{j-1}{n}
\end{aligned}
$$

Therefore, in view of (2.2) and (2.3), we get

$$
\begin{aligned}
0 & \leq U_{n, j}\left(x e_{0}-e_{1} ; x\right)=\sum_{k=1}^{j-1} p_{n, k}(x) \frac{k}{n}+\sum_{k=j}^{n-1} p_{n, k}(x)\left(\frac{k}{n}-a_{n, k}\right) \\
& \leq \sum_{k=1}^{j-1} p_{n, k}(x) \frac{j-1}{n}+\sum_{k=j}^{n-1} p_{n, k}(x) \frac{j-1}{n} \leq \frac{j-1}{n} \sum_{k=0}^{n} p_{n, k}(x)=\frac{j-1}{n} .
\end{aligned}
$$

b) Taking into account the inequality $(a+b)^{2} \leq 2\left(a^{2}+b^{2}\right),(2.3)$ and Lemma 2.1, we find that

$$
\begin{aligned}
& U_{n, j}\left(\left(e_{1}-x e_{0}\right)^{2} ; x\right)=\sum_{k=0}^{n} p_{n, k}(x)\left(a_{n, k}-x\right)^{2} \\
& \leq 2 \sum_{k=0}^{n} p_{n, k}(x)\left(a_{n, k}-\frac{k}{n}\right)^{2}+2 \sum_{k=0}^{n} p_{n, k}(x)\left(\frac{k}{n}-x\right)^{2} \\
& \quad=2 \sum_{k=1}^{j-1} p_{n, k}(x)\left(\frac{k}{n}\right)^{2}+2 \sum_{k=j}^{n-1} p_{n, k}(x)\left(a_{n, k}-\frac{k}{n}\right)^{2}+\frac{2}{n} x(1-x) \\
& \leq 2\left(\frac{j-1}{n}\right)^{2} \sum_{k=1}^{j-1} p_{n, k}(x)+2\left(\frac{j-1}{n}\right)^{2} \sum_{k=j}^{n-1} p_{n, k}(x)+\frac{2}{n} x(1-x) \\
& =2\left(\frac{j-1}{n}\right)^{2}\left(1-(1-x)^{n}-x^{n}\right)+\frac{2}{n} x(1-x) \leq 2\left(\frac{j-1}{n}\right)^{2} n x(1-x)+\frac{2}{n} x(1-x) \\
& =\frac{2}{n}\left((j-1)^{2}+1\right) \varphi^{2}(x) .
\end{aligned}
$$


c) In view of $(a+b)^{4} \leq 8\left(a^{4}+b^{4}\right),(2.3)$ and $\sum_{k=0}^{n}(k-n x)^{4} p_{n, k}(x)=3 n^{2} \varphi^{4}(x)+n\left(\varphi^{2}(x)-6 \varphi^{4}(x)\right)$, we obtain

$$
\begin{aligned}
& U_{n, j}\left(\left(e_{1}-x e_{0}\right)^{4} ; x\right)=\sum_{k=0}^{n} p_{n, k}(x)\left(a_{n, k}-x\right)^{4} \\
& \leq 8 \sum_{k=0}^{n} p_{n, k}(x)\left(a_{n, k}-\frac{k}{n}\right)^{4}+8 \sum_{k=0}^{n} p_{n, k}(x)\left(\frac{k}{n}-x\right)^{4} \\
& =8 \sum_{k=1}^{j-1} p_{n, k}(x)\left(\frac{k}{n}\right)^{4}+8 \sum_{k=j}^{n-1} p_{n, k}(x)\left(a_{n, k}-\frac{k}{n}\right)^{4}+\frac{8}{n^{4}} \sum_{k=0}^{n} p_{n, k}(x)(k-n x)^{4} \\
& \leq 8\left(\frac{j-1}{n}\right)^{4} \sum_{k=1}^{j-1} p_{n, k}(x)+8\left(\frac{j-1}{n}\right)^{4} \sum_{k=j}^{n-1} p_{n, k}(x) \\
& \quad+\frac{8}{n^{4}}\left(3 n^{2} \varphi^{4}(x)+n\left(\varphi^{2}(x)-6 \varphi^{4}(x)\right)\right) \\
& \leq \quad 8\left(\frac{j-1}{n}\right)^{4}+\frac{8}{n^{4}} 4 n^{2} \varphi^{2}(x) \leq 8\left(\frac{j-1}{n}\right)^{4}+\frac{8}{n^{2}} \leq \frac{8}{n^{2}}\left((j-1)^{4}+1\right) .
\end{aligned}
$$

This completes the proof of the lemma.

The main result is the following theorem.

Theorem 2.1. Let $U_{n, j}$ be given by (1.3)-(1.4). Then there exists $C_{2}>0$ depending only on $j$ such that

$$
\begin{aligned}
\mid n\left(U_{n, j}(f ; x)-f(x)\right) & +f^{\prime}(x) n U_{n, j}\left(x e_{0}-e_{1} ; x\right)-\frac{1}{2} f^{\prime \prime}(x) n U_{n, j}\left(\left(e_{1}-x e_{0}\right)^{2} ; x\right) \mid \\
& \leq C_{2} \omega_{\varphi}^{1}\left(f^{\prime \prime} ; \frac{1}{\sqrt{n}}\right)
\end{aligned}
$$

for all $x \in[0,1], f \in C^{2}[0,1]$ and $n \geq j \geq 2$. Furthermore

$$
0 \leq \liminf _{n \rightarrow \infty} n U_{n, j}\left(x e_{0}-e_{1} ; x\right) \leq \limsup _{n \rightarrow \infty} n U_{n, j}\left(x e_{0}-e_{1} ; x\right) \leq j-1
$$

and

$$
\begin{aligned}
0 & \leq \liminf _{n \rightarrow \infty} n U_{n, j}\left(\left(e_{1}-x e_{0}\right)^{2} ; x\right) \\
& \leq \limsup _{n \rightarrow \infty} n U_{n, j}\left(\left(e_{1}-x e_{0}\right)^{2} ; x\right) \leq \frac{1}{2}\left((j-1)^{2}+1\right) .
\end{aligned}
$$

Proof. Because $U_{n, j}(f ; 0)=f(0)$ and $U_{n, j}(f ; 1)=f(1)$, the estimate (2.4) is satisfied for $x \in$ $\{0,1\}$. Now let $x \in(0,1)$ and $t \in[0,1]$. By Taylor's formula, we have

$$
f(t)=f(x)+f^{\prime}(x)(t-x)+\frac{1}{2} f^{\prime \prime}(x)(t-x)^{2}+\int_{x}^{t}\left(f^{\prime \prime}(u)-f^{\prime \prime}(x)\right)(t-u) d u .
$$




\section{Hence}

$$
\begin{aligned}
& \left|U_{n, j}(f ; x)-f(x)+f^{\prime}(x) U_{n, j}\left(x e_{0}-e_{1} ; x\right)-\frac{1}{2} f^{\prime \prime}(x) U_{n, j}\left(\left(e_{1}-x e_{0}\right)^{2} ; x\right)\right| \\
& \quad=\left|U_{n, j}\left(\int_{x}^{t}\left(f^{\prime \prime}(u)-f^{\prime \prime}(x)\right)(t-u) d u ; x\right)\right| \\
& \quad \leq U_{n, j}\left(\left|\int_{x}^{t}\right| f^{\prime \prime}(u)-f^{\prime \prime}(x)|| t-u|d u| ; x\right) .
\end{aligned}
$$

On the other hand

$$
\left|\int_{x}^{u} \frac{d v}{\varphi(v)}\right| \leq \varphi^{-1}(x)|u-x|^{1 / 2}\left|\int_{x}^{u} \frac{d v}{|u-v|^{1 / 2}}\right| \leq 2 \varphi^{-1}(x)|u-x|, \quad x \in(0,1), u \in[0,1]
$$

(cf. [2, Lemma 9.6.1]). Hence, for all $g \in W(\varphi)$, we have

$$
\begin{aligned}
\left|\int_{x}^{t}\right| f^{\prime \prime}(u)-f^{\prime \prime}(x)|| t-u|d u| & \\
\leq & \left|\int_{x}^{t}\right| f^{\prime \prime}(u)-g(u)|| t-u|d u|+\left|\int_{x}^{t}\right| g(u)-g(x)|| t-u|d u| \\
& +\left|\int_{x}^{t}\right| g(x)-f^{\prime \prime}(x)|| t-u|d u| \\
\leq & \frac{1}{2}(t-x)^{2}\left\|f^{\prime \prime}-g\right\|+\left|\int_{x}^{t}\right| \int_{x}^{u} g^{\prime}(v) d v|| t-u|d u|+\frac{1}{2}(t-x)^{2}\left\|f^{\prime \prime}-g\right\| \\
\leq & (t-x)^{2}\left\|f^{\prime \prime}-g\right\|+\left\|\varphi g^{\prime}\right\|\left|\int_{x}^{t}\right| \int_{x}^{u} \frac{d v}{\varphi(v)}|| t-u|d u| \\
\leq & (t-x)^{2}\left\|f^{\prime \prime}-g\right\|+2 \varphi^{-1}(x)\left\|\varphi g^{\prime}\right\|\left|\int_{x}^{t}\right| u-x \| t-u|d u| \\
\leq & (t-x)^{2}\left\|f^{\prime \prime}-g\right\|+2 \varphi^{-1}(x)|t-x|^{3}\left\|\varphi g^{\prime}\right\| .
\end{aligned}
$$

\section{Combining (2.7), (2.8), Hölder's inequality and Lemma 2.2, we get}

$$
\begin{aligned}
& \left|U_{n, j}(f ; x)-f(x)+f^{\prime}(x) U_{n, j}\left(x e_{0}-e_{1} ; x\right)-\frac{1}{2} f^{\prime \prime}(x) U_{n, j}\left(\left(e_{1}-x e_{0}\right)^{2} ; x\right)\right| \\
& \leq\left\|f^{\prime \prime}-g\right\| U_{n, j}\left(\left(e_{1}-x e_{0}\right)^{2} ; x\right)+2 \varphi^{-1}(x)\left\|\varphi g^{\prime}\right\| U_{n, j}\left(\left|e_{1}-x e_{0}\right|^{3} ; x\right) \\
& \leq\left\|f^{\prime \prime}-g\right\| U_{n, j}\left(\left(e_{1}-x e_{0}\right)^{2} ; x\right) \\
& +2 \varphi^{-1}(x)\left\|\varphi g^{\prime}\right\|\left(U_{n, j}\left(\left(e_{1}-x e_{0}\right)^{2} ; x\right)\right)^{1 / 2}\left(U_{n, j}\left(\left(e_{1}-x e_{0}\right)^{4} ; x\right)\right)^{1 / 2} \\
& \leq \frac{2}{n}\left((j-1)^{2}+1\right) \varphi^{2}(x)\left\|f^{\prime \prime}-g\right\| \\
& +2 \varphi^{-1}(x)\left\|\varphi g^{\prime}\right\| \sqrt{\frac{2}{n}\left((j-1)^{2}+1\right)} \varphi(x) \frac{2 \sqrt{2}}{n} \sqrt{(j-1)^{4}+1} \\
& \leq \frac{8}{n} \sqrt{(j-1)^{2}+1} \sqrt{(j-1)^{4}+1}\left(\left\|f^{\prime \prime}-g\right\|+\frac{1}{\sqrt{n}}\left\|\varphi g^{\prime}\right\|\right) .
\end{aligned}
$$


Taking the infimum on the right hand side over all $g \in W(\varphi)$, we find

$$
\begin{aligned}
& \left|n\left(U_{n, j}(f ; x)-f(x)\right)+f^{\prime}(x) n U_{n, j}\left(x e_{0}-e_{1} ; x\right)-\frac{1}{2} f^{\prime \prime}(x) n U_{n, j}\left(\left(e_{1}-x e_{0}\right)^{2} ; x\right)\right| \\
& \leq 8 \sqrt{(j-1)^{2}+1} \sqrt{(j-1)^{4}+1} K_{1, \varphi}\left(f ; \frac{1}{\sqrt{n}}\right) .
\end{aligned}
$$

Hence, by (1.6), we obtain the estimation (2.4).

Finally, the estimations (2.5) follow from Lemma 2.2, a). Again, due to Lemma 2.2, b), we obtain

$$
n U_{n, j}\left(\left(e_{1}-x e_{0}\right)^{2} ; x\right) \leq 2\left((j-1)^{2}+1\right) \varphi^{2}(x) \leq \frac{1}{2}\left((j-1)^{2}+1\right) .
$$

Hence we find the estimations (2.6), which completes the proof of the theorem.

Corollary 2.1. There exists $C_{3}>0$ such that

$$
\left|n\left(U_{n, 2}(f ; x)-f(x)\right)+\left(f^{\prime}(x)-x f^{\prime \prime}(x)\right) n U_{n, 2}\left(x e_{0}-e_{1} ; x\right)\right| \leq C_{3} \omega_{\varphi}^{1}\left(f^{\prime \prime} ; \frac{1}{\sqrt{n}}\right)
$$

for all $x \in[0,1], f \in C^{2}[0,1]$ and $n \geq 2$. Furthermore

$$
0 \leq \liminf _{n \rightarrow \infty} n U_{n, 2}\left(x e_{0}-e_{1} ; x\right) \leq \limsup _{n \rightarrow \infty} n U_{n, 2}\left(x e_{0}-e_{1} ; x\right) \leq 1 .
$$

Proof. It follows immediately from Theorem 2.1, taking into account that $U_{n, 2}\left(e_{0} ; x\right)=1$, $U_{n, 2}\left(e_{2} ; x\right)=x^{2}$ and

$$
\begin{aligned}
U_{n, 2}\left(\left(e_{1}-x e_{0}\right)^{2} ; x\right) & =U_{n, 2}\left(e_{2} ; x\right)-2 x U_{n, 2}\left(e_{1} ; x\right)+x^{2} U_{n, 2}\left(e_{0} ; x\right) \\
& =2 x\left(x-U_{n, 2}\left(e_{1} ; x\right)\right)=2 x U_{n, 2}\left(x e_{0}-e_{1} ; x\right) .
\end{aligned}
$$

\section{REFERENCES}

[1] J. M. Aldaz, O. Kounchev and H. Render: Shape preserving properties of generalized Bernstein operators on extended Chebyshev spaces. Numer. Math. 114 (2009), 1-25.

[2] Z. Ditzian and V. Totik: Moduli of Smoothness. Springer, New York, 1987.

[3] Z. Finta: On generalized Voronovskaja theorem for Bernstein polynomials. Carpathian J. Math. 28 (2012), 231-238.

[4] M. S. Floater: On the convergence of derivatives of Bernstein approximation. J. Approx. Theory. 134 (2005), 130-135.

[5] H. Gonska and I. Raşa: Asymptotic behavior of differentiated Bernstein polynomials. Math. Vesnik. 61 (2009), 53-60.

[6] H. Gonska and G. Tachev: A quantitative variant of Voronovskaja's theorem. Result. Math. 53 (2009), 287-294.

[7] H. Gonska, M. Heilmann and I. Raşa: Asymptotic behavior of differentiated Bernstein polynomials revisited. General Math. 18 (2010), 45-53.

[8] J. P. King: Positive linear operators which preserve $x^{2}$. Acta Math. Hungar. 99 (2003), 203-208.

\section{BABEŞ-BOLYAI UNIVERSITY}

DEPARTMENT OF MATHEMATICS

STR M. KOGĂLNICEANU No 1, 400084, CluJ-NAPOCA, RoMANIA

Email address: fzoltan@math.ubbcluj.ro 